\title{
Plutôt jeune, riche et en bonne santé...
}

\author{
Anna Sax \\ Lic. oec. publ., MHA, membre de la rédaction
}

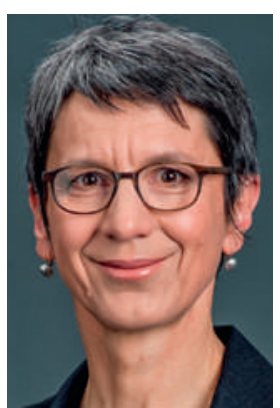

J'ai récemment rempli une fois de plus un questionnaire sur un portail Internet pour déterminer mon espérance de vie. Les questions portaient notamment sur les facteurs héréditaires, le comportement de santé, la formation, les revenus et les contacts sociaux. Et là, j'ai pu (dû) constater que mon espérance de vie était de 98 ans. Près d'un siècle! Je retournai ensuite sur le portail et corrigeai trois facteurs: formation, revenus, état civil. Selon la nouvelle version du questionnaire, je n'ai pas fait d'études secondaires, je gagne moins de 48000 francs par an et je suis divorcée. Et surprise, mon espérance de vie n'est "plus que» de 89 ans selon les probabilités statistiques. Si, en plus, je deviens fumeuse, prends quelques kilos et bois un ou deux verres de plus par jour, le logiciel me retire des années de vie supplémentaires. Ce test est contestable, parce que de telles valeurs statistiques ont certes un intérêt du point de vue épidémiologique ou dans le domaine des assurances, mais ne peuvent être appliquées à un cas isolé. Ce qui est intéressant, c'est qu'il montre ce que l'on sait depuis longtemps et que le dernier Rapport sur la santé confirme une fois de plus: être riche et cultivé est bénéfique pour la santé. Les universitaires bien payés fument moins, mangent plus sainement, bougent plus, ont moins de kilos superflus, sont mieux intégrés socialement, plus heureux et en meilleure santé. Et ils vivent plus vieux.

\section{La situation sociale a bien plus d'influence sur la santé que la médecine.}

La situation sociale a bien plus d'influence sur la santé que la médecine. D'un point de vue économique, investir dans la sécurité sociale et la compétence de santé est plus efficace que d'introduire de plus en plus de techniques médicales et de médicaments coûteux. Ce n'est pas nouveau et de nombreux rapports et études, parmi lesquels le Rapport sur la santé 2015 susmentionné, qui porte sur l'apparition et la lutte contre les maladies non transmissibles, le confirment. La connais- sance de l'importance des déterminants sociaux de la santé est empiriquement fondée et ancrée de longue date dans les milieux de santé publique, mais ne joue pratiquement aucun rôle au niveau politique et sur le marché de la santé: qui voudrait investir dans un élément qui contribuerait, au final, à réduire les recettes des hôpitaux, du corps médical et de l'industrie sanitaire? Certes, la Confédération et les cantons mettent actuellement au point une stratégie contre les maladies non transmissibles qui évoque également la possibilité d'améliorer la situation des couches de la population peu privilégiées. Mais à condition que cela ne coûte rien au contribuable.

\section{Instaurer une égalité des chances n'est pas un} modèle commercial et il n'existe pas de marché pour la transmission de compétences de santé.

Malheureusement, même celles et ceux qui jouissent d'une bonne situation n'échappent pas au vieillissement et à la mort. La peur du déclin physique et mental imminent est très présente chez la génération des babyboomers, dont je fais partie. Les affaires de tous ceux qui ont quelque chose à proposer à ce public cible - programmes de fitness pour le corps et l'esprit, traitements antirides, offres de bien-être, alicaments, culture et formation - s'annoncent donc excellentes. La publicité montre de plus en plus des personnes «âgées» en pleine forme, quoique je les soupçonne fortement d'être des actrices et acteurs plus jeunes, aux cheveux teints en gris. A l'inverse, instaurer une égalité des chances n'est pas un modèle commercial et il n'existe pas de marché pour la transmission de compétences de santé. Les personnes vraiment âgées et fragiles sont rarement présentées comme des modèles. Malgré l'allongement de notre espérance de vie, il est de plus en plus difficile de vieillir et de mourir dignement. Par conséquent, l'idée que je pourrais peut-être vivre encore plus de 40 ans ne suscite pas, chez moi non plus, un enthousiasme sans mélange. 